\title{
Three-Step Telescoped Synthesis of Monosubstituted Vicinal Diamines from Aldehydes
}

\author{
Lledó Bou-Iserte, Antonio Latorre, Santiago Rodríguez, and Florenci V. González*(i) \\ Departament de Química Inorgànica i Orgànica, Universitat Jaume I, Castelló 12071, Spain \\ Supporting Information
}

ABSTRACT: Aldehydes are easily transformed into vicinal diamines and piperazines through a one-pot procedure including a Darzens reaction and treatment with an amine or diamine and then with a reducing agent. Additionally, quinoxalines can be accessed by reaction with 1,2-benzenediamine under oxidative conditions. These transformations are simple methods for the preparation of synthetically interesting monosubstituted diamines, piperazines, and quinoxalines.

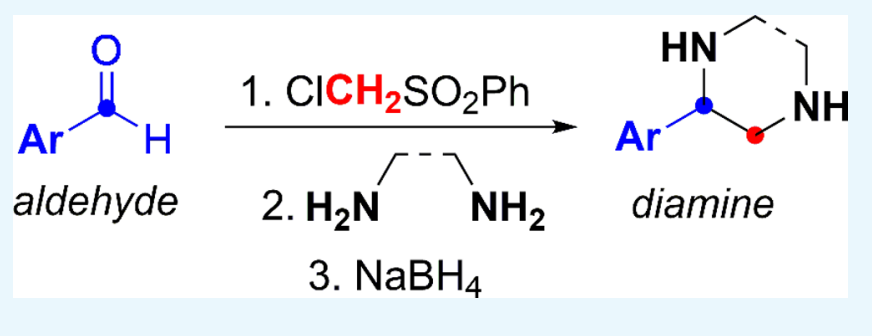

\section{INTRODUCTION}

Vicinal diamines are important building blocks found in many bioactive molecules (Figure 1), ${ }^{1}$ natural products, ${ }^{2}$ and metal complexes displaying interesting properties. ${ }^{3}$ Also, quinoxalines and piperazines have interesting medical properties. For instance, quinoxaline derivatives display anticancer, ${ }^{4}$ antiviral, ${ }^{5}$ antibacterial, ${ }^{6}$ anti-inflammatory, ${ }^{7}$ and antibiotic ${ }^{8}$ activities. A piperazine moiety is considered a "privileged scaffold" in medicinal chemistry ${ }^{9}$ and is present in many biologically active molecules.

Monosubstituted vicinal diamines are present in potent bioactive compounds such as tetracyclic antidepressants ${ }^{1}$ mianserin and mirtazapine or k-opioid antagonist ICI$199441^{1 \mathrm{e}}$ (Figure 1A). Although interesting approaches have been recently reported for the preparation of monosubstituted $^{10}$ and disubstituted ${ }^{11}$ diamines, more approaches are welcome.

We report herein the preparation of monosubstituted diamines from aldehydes in a one-pot three-step process. The process comprises first the formation of an epoxysulfone by a Darzens reaction between an aldehyde and chloromethylphenylsulfone and then addition of an amine attacking the $\beta$ position of the epoxide, affording an $\alpha$-aminoaldehyde, which upon reductive amination with a second amine gives the corresponding diamine (Figure 1B). This chemical transformation is inspired by our previous work about chemical transformations of nitroepoxides. ${ }^{11}$

\section{RESULTS AND DISCUSSION}

We first prepared epoxysulfone 1a through a Darzens reaction between benzaldehyde and chloromethylphenylsulfone. Then, epoxysulfone 1a was combined with benzylamine in dichloromethane (DCM) for $8 \mathrm{~h}$ at room temperature, and then sodium borohydride was added. The reaction worked satisfactorily, affording diamine $\mathbf{2 a}$ (Scheme 1). However, the conversion of epoxysulfone 1a into diamine 2 a was very low yielding.
Hence, we turned our attention to a one-pot procedure for the transformation of aldehydes into diamines by combining the four reactions shown in Scheme 1. One-pot procedures represent interesting synthetic approaches, ${ }^{12}$ which might improve yields as compared to step-wise sequences. First, benzaldehyde was reacted with chloromethylphenylsulfone previously treated with sodium tert-butoxide in dichloromethane. After the mixture was stirred for $1 \mathrm{~h}$ at $0{ }^{\circ} \mathrm{C}$ and then for $1 \mathrm{~h}$ at room temperature, benzylamine ( 3 equiv.) was added, and the resulting mixture was stirred for $8 \mathrm{~h}$ at room temperature and then sodium borohydride (6 equiv.) was added and stirred again for $8 \mathrm{~h}$. Under these experimental conditions, diamines were obtained but chemical yields were not satisfying (Table 1, entries 4, 7, and 9). Under these conditions, the corresponding benzyl $p$-methylbenzyl amine was also isolated when using $p$-tolualdehyde, denoting an undesired attack of the benzylamine on the epoxysulfone (Scheme 2).

To increase the yield, an optimization process was performed by modifying the temperature, solvent, and number of equivalents. Higher yields were obtained when a temperature of $0{ }^{\circ} \mathrm{C}$ was kept during the amine treatment, favoring the attack of the amine at the $\beta$-position. This slowed the reaction, and the duration for that step had to be increased up to $18 \mathrm{~h}$. Optimized conditions also comprise an increase of the number of equivalents of amine and sodium borohydride (4 and 8 , respectively). A screening of solvents gave 1,2dichloroethane as the best option in all cases (entries $1,5,8$, and 13). Probably, the improvement of the yield with 1,2dichloroethane as compared to dichloromethane is due to its lower volatility avoiding losses of solvents after such a long reaction time. Other assayed conditions such as the use of lithium chloride as an additive and tetrahydrofuran (THF) as a

Received: November 4, 2018

Accepted: January 16, 2019

Published: January 29, 2019 
A)

Examples of biologically active diamines<smiles>CN(C(=O)Cc1ccc(Cl)c(Cl)c1)C(CN1CCCC1)c1ccccc1</smiles><smiles>[X]c1ccccc1CN1CCN(C)CC1c1ccccc1</smiles>

ICl-199441

$\mathrm{X}=\mathrm{CH}$, Mianserin $\mathrm{X}=\mathrm{N}$, Mirtazapine

B) One-pot three-step sequence from aldehyde to diamine

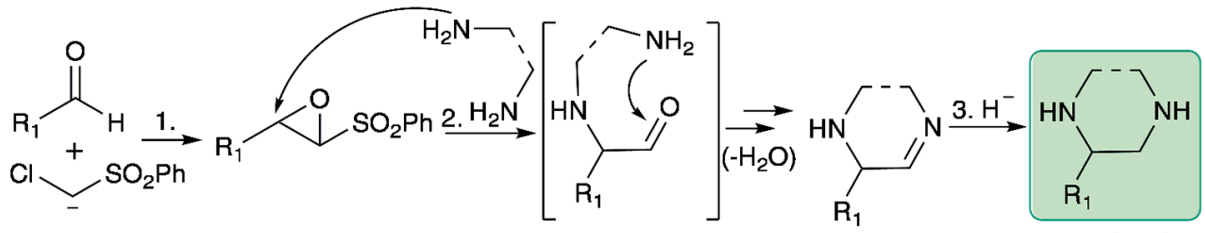

-monosubstituted

diamines

Figure 1. (a) Examples of diamines. (b) Reaction design.

Scheme 1. Synthesis of Diamines through Epoxysulfones

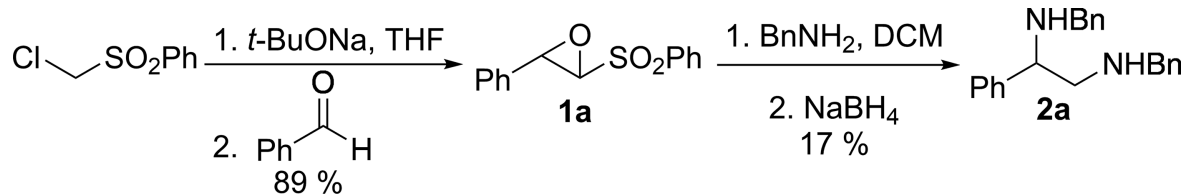

Table 1. Synthesis of Diamines from Aldehydes ${ }^{a}$

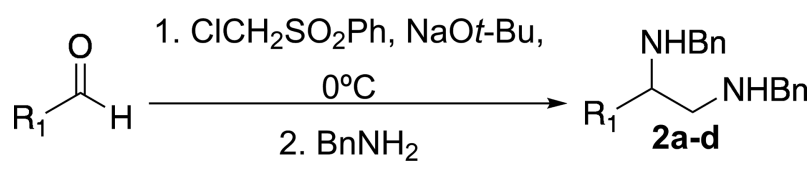

3. $\mathrm{NaBH}_{4}$

\begin{tabular}{clclc} 
entry & \multicolumn{1}{c}{$\mathrm{R}^{1}$} & diamine & solvent & yield $(\%)^{b}$ \\
1 & $\mathrm{Ph}$ & $\mathbf{2 a}$ & DCE & 44 \\
2 & $p$ Me-Ph & $\mathbf{2 b}$ & DCM & 15 \\
3 & $p$ Me-Ph & $\mathbf{2 b}$ & THF & 34 \\
4 & $p$ Me-Ph & $\mathbf{2 b}$ & DCE & $24^{c}$ \\
5 & $p$ Me-Ph & $\mathbf{2 b}$ & DCE & 40 \\
6 & $p$ Cl-Ph & $\mathbf{2 c}$ & DCM & 25 \\
7 & $p$ Cl-Ph & $\mathbf{2 c}$ & DCE & $22^{c}$ \\
8 & $p$ Cl-Ph & $\mathbf{2 c}$ & DCE & 52 \\
9 & $p$ Cl-Ph & $\mathbf{2 c}$ & THF & $4^{d}$ \\
10 & $p$ Cl-Ph & $\mathbf{2 c}$ & DCE & $23^{e}$ \\
11 & $p C l-P h$ & $2 c$ & DCE & $5^{f}$ \\
12 & $p$ F-Ph & $\mathbf{2 d}$ & DCE & $26^{c}$ \\
13 & $p F-P h$ & $\mathbf{2 d}$ & DCE & 30 \\
\hline
\end{tabular}

${ }^{a}$ Reactions were performed using benzylamine (4 equiv.) and sodium borohydride ( 8 equiv.) at $0{ }^{\circ} \mathrm{C}$. ${ }^{b}$ Yield of the isolated product. ${ }^{c}$ Reactions were carried out at room temperature. ${ }^{d}$ The reaction was carried out by adding lithium chloride (10 equiv.). ${ }^{e}$ The reaction was carried out under phase-transfer conditions with aq. $\mathrm{NaOH}(50 \%)(1$ $\mathrm{mL} / \mathrm{mmol}$ ) and tetra- $n$-butyl ammonium bromide (0.2 equiv.). $f_{\text {Sodium triacetoxyborohydride (8 equiv.) was used instead of sodium }}$ borohydride. solvent (entry 9), phase-transfer conditions (entry 10), or the use of sodium triacetoxyborohydride instead of sodium borohydride (entry 11) did not improve the yield.

Aliphatic aldehydes (propionaldehyde and isobutyraldehyde) and ortho-substituted aromatic aldehydes (2-chlorobenzaldehyde) were also assayed under different conditions, but only traces of desired diamines were detected. Then, we assayed the use of two different amines during the process instead of only one. Thus, $N$-benzylmethylamine (4 equiv.) was added first, then benzylamine (4 equiv.) was added when TLC showed total consumption of epoxysulfone and finally sodium borohydride. Following this procedure, diamines $\mathbf{2 e - h}$ were prepared (Scheme 3). However, when benzaldehyde was first treated with benzylamine and $\mathrm{N}$-benzylmethylamine was then added, diamine $\mathbf{2 a}$ was obtained. Presumably, the aminoaldehyde intermediate resulting from the opening of epoxysulfone by benzylamine (see Figure $1 \mathrm{~b}$ ) reacts with a second equivalent of benzylamine to give a corresponding imine in contrast with $N$-benzylmethylamine. The same experimental procedure was also used for diamines $\mathbf{2 e - h}$, using morpholine instead of $N$-benzylmethylamine, and only traces of desired diamines were detected.

Then, we extended the study to the use of other amines different from benzylamines. When optimized experimental conditions (Table 1 ) were applied but $n$-butylamine was used as a starting material instead of benzylamine, the chemical yield of desired diamines was very low, even when the reaction was performed at room temperature or at reflux. To activate epoxysulfone for the amine attack, Lewis acids were added to the reaction mixture after epoxysulfone formation. Magnesium bromide, magnesium sulfate, and titanium isopropoxide had no 
Scheme 2. Formation of Secondary Amines

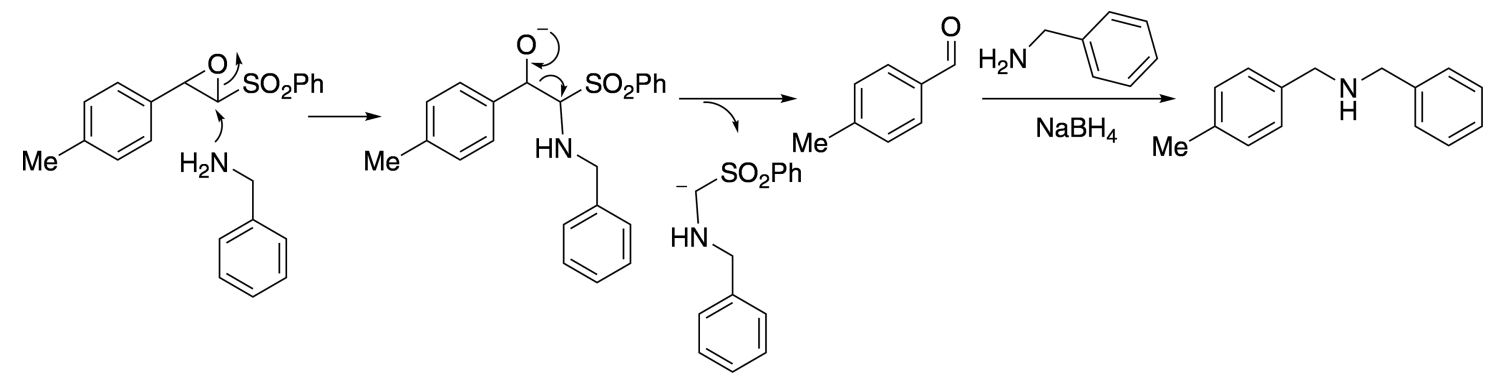

Scheme 3. Synthesis of Differently Substituted Diamines from Aldehydes

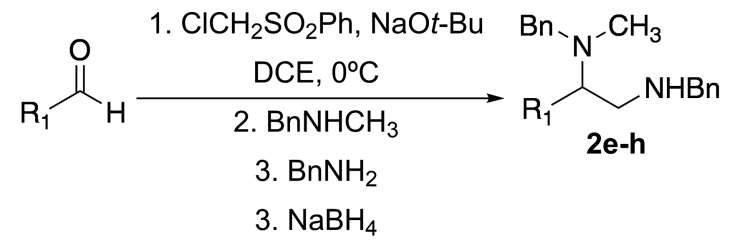<smiles>CN(Cc1ccccc1)C(CNc1ccccc1)c1ccccc1</smiles><smiles>Cc1ccc(C([18Br])N(C)Cc2ccccc2)cc1</smiles><smiles>CN(Cc1ccccc1)C(CNc1ccccc1)c1ccc(Cl)cc1</smiles><smiles>CN(Cc1ccccc1)C(N[18OH])c1ccc(F)cc1</smiles>

effect on the reactivity of epoxysulfones. However, the addition of lithium chloride (10 equiv.) afforded the desired diamines $\mathbf{2 i}-\mathbf{j}$ (Scheme 4). Tetrahydrofuran gave higher yields than dichloroethane probably because of the higher solubility of lithium chloride.

Scheme 4. Synthesis of Diamines from Aliphatic Amines

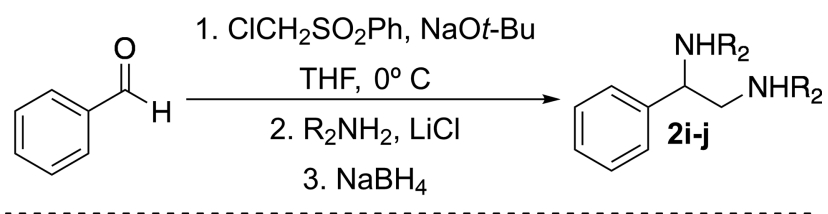<smiles>CCCCNC(=[18O])C(NCCCC)c1ccccc1</smiles><smiles>c1ccc(CCCNCC(CNCCc2ccccc2)c2ccccc2)cc1</smiles>

Cyclic diamines were also prepared. Piperazines $\mathbf{3 a}$ and $\mathbf{3 b}$ were prepared following the same experimental conditions as those for diamines starting from benzaldehyde and tolualdehyde, respectively (Scheme 5). Quinoxaline 4 was prepared using 1,2-benzenediamine and in the presence of air ${ }^{13}$ (Scheme 5).
Scheme 5. Synthesis of Cyclic Diamines

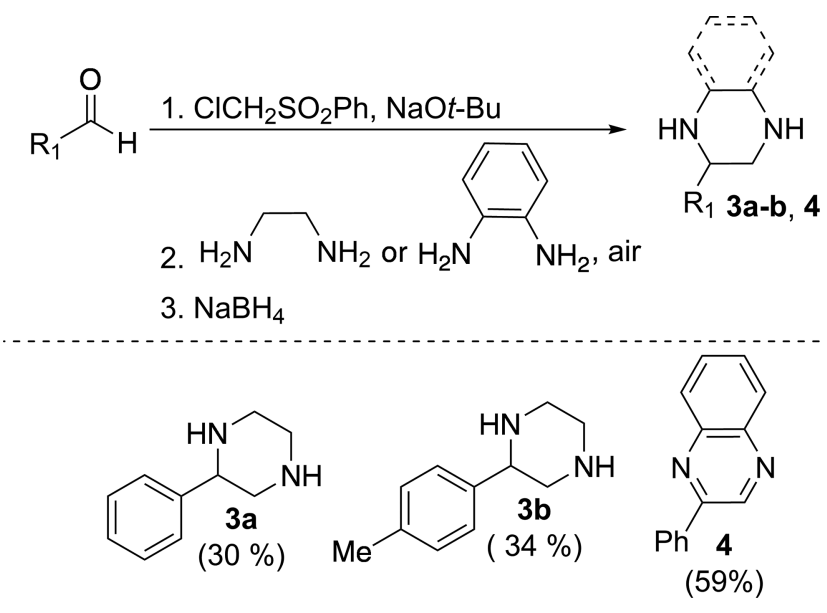

\section{CONCLUSIONS}

In summary, we reported herein that 1,2-diamines, piperazines, and quinoxalines can be prepared starting from aldehydes. It is a one-pot procedure through three steps: A Darzens reaction with chloromethylsulfone and sodium tert-butoxide, treatment with an excess of amines or diamines, and reductive treatment to afford corresponding diamines. Also, quinoxalines can be prepared in a similar manner but using 1,2-benzenediamine upon oxidative conditions. Further research related to the synthetic use of epoxysulfones is going on in our lab and will be reported in the near future.

\section{EXPERIMENTAL SECTION}

General Information. All reactions were carried out under a nitrogen atmosphere with magnetic stirring, unless otherwise specified. Used reagents and solvents were obtained from commercial sources and were purified accordingly before use. EM Science Silica Gel 60 was used for column chromatography, whereas TLC was performed with precoated plates (Kieselgel 60, $\mathrm{F}_{254}, 0.25 \mathrm{~mm}$ ). ${ }^{1} \mathrm{H}$ NMR spectra and ${ }^{13} \mathrm{C}$ NMR spectra were recorded in $\mathrm{CDCl}_{3}\left({ }^{1} \mathrm{H}, 7.24 \mathrm{ppm} ;{ }^{13} \mathrm{C} 77.0 \mathrm{ppm}\right)$ solution at $30{ }^{\circ} \mathrm{C}$ on a $300 \mathrm{MHz}$ or a $400 \mathrm{MHz}$ NMR spectrometer. Mass spectra were recorded on a QTOF I (quadrupole-hexapole-TOF) mass spectrometer with an orthogonal Z-spray-electrospray interface.

General Experimental Procedure for the Synthesis of Epoxysulfone 1a. $t$-BuONa ( $1.1 \mathrm{mmol}, 1.1$ equiv.) was slowly added to a cold $\left(0{ }^{\circ} \mathrm{C}\right)$ stirred solution of chloromethyl phenyl sulfone ( $1.1 \mathrm{mmol}, 1.1$ equiv.) in THF $(5 \mathrm{~mL})$. The mixture was stirred for $15 \mathrm{~min}$, followed by dropwise addition of a solution of the corresponding aldehyde ( $1 \mathrm{mmol}, 1$ equiv.) 
in THF $(5 \mathrm{~mL})$. The reaction mixture was then stirred for 30 min and monitored by TLC. Quenching was done by adding ammonium chloride saturated aqueous solution $(10 \mathrm{~mL})$, and then the mixture was allowed to warm to room temperature before being extracted with ethyl ether $(3 \times 10 \mathrm{~mL})$. The organic layers were washed with $1 \mathrm{M}$ hydrochloric acid (10 $\mathrm{mL}$ ), then with sodium bicarbonate saturated aqueous solution $(10 \mathrm{~mL})$, and finally with brine; then, the layers were dried using $\mathrm{Na}_{2} \mathrm{SO}_{4}$ and concentrated under vacuum. The crude material was purified using chromatography (silica gel, hexanes/ethyl acetate $(9: 1$ to $7: 3))$ to give the pure compound.

2-Phenyl-3-(phenylsulfonyl)oxirane (1a). White crystals, mp 105-106 ${ }^{\circ} \mathrm{C}$ (yield $\left.231 \mathrm{mg}, 89 \%\right):{ }^{1} \mathrm{H}$ NMR (400 MHz, $\left.\mathrm{CDCl}_{3}\right) \delta 7.96-7.89(\mathrm{~m}, 2 \mathrm{H}), 7.70-7.63(\mathrm{~m}, 1 \mathrm{H}), 7.61-7.51$ (m, 2H), 7.33-7.26 (m, 3H), 7.22-7.19 (m, 2H), $4.52(\mathrm{~d}, J=$ $1.5 \mathrm{~Hz}, 1 \mathrm{H}), 4.11(\mathrm{~d}, J=1.6 \mathrm{~Hz}, 1 \mathrm{H}) \mathrm{ppm} ;{ }^{13} \mathrm{C} \mathrm{NMR}(101$ $\left.\mathrm{MHz}, \mathrm{CDCl}_{3}\right) \delta 136.94,134.58,132.72,129.60,129.50$, 128.88, 128.82, 126.10, 71.05, 57.48 ppm; HRMS (ESI) $\mathrm{m} / z$ calcd for $\mathrm{C}_{14} \mathrm{H}_{12} \mathrm{O}_{3} \mathrm{~S}[\mathrm{M}+\mathrm{H}]^{+}:$261.0585, found: 261.0582; IR $(\mathrm{KBr}) \nu 3313,3060,3025,2920,2830,1757,1676,1513$, 1495, 1454, 1119, 818, 740, $700 \mathrm{~cm}^{-1}$.

General Experimental Procedure for the Synthesis of Diamines. To an ice-cold, stirred solution of the corresponding epoxysulfone ( $1 \mathrm{mmol}, 1$ equiv.) in DCM $(10 \mathrm{~mL})$, the corresponding amine ( $3 \mathrm{mmol}, 3$ equiv.) was slowly added. The reaction mixture was stirred for $8 \mathrm{~h}$, and then $\mathrm{NaBH}_{4}(6$ mmol, 6 equiv.) was added portionwise. The reaction mixture was then stirred for $1.5 \mathrm{~h}$ and was monitored by TLC. Then, it was quenched by ammonium chloride saturated aqueous solution $(10 \mathrm{~mL})$ and was allowed to warm to room temperature before being extracted with DCM $(3 \times 10 \mathrm{~mL})$. The organic layers resulting from the extraction were washed with $1 \mathrm{M}$ hydrochloric acid $(10 \mathrm{~mL})$, then with sodium bicarbonate saturated aqueous solution $(10 \mathrm{~mL})$, and finally with brine; then, these layers were dried using $\mathrm{Na}_{2} \mathrm{SO}_{4}$ and concentrated under vacuum. The resulting crude material was purified by chromatography (silica gel, hexanes/ethyl acetate ( $7: 3$ to $1: 1)$ ) to give the desired pure compound.

Method A. $t$-BuONa (1.2 mmol, 1.2 equiv.) was added to a $0{ }^{\circ} \mathrm{C}$ cold solution of chloromethyl phenyl sulfone $(1.2 \mathrm{mmol}$, 1.2 equiv. $)$ in DCE $(5 \mathrm{~mL})$. The resulting mixture was stirred for $15 \mathrm{~min}$, and then the corresponding aldehyde $(1 \mathrm{mmol}, 1$ equiv.) dissolved in DCE ( $5 \mathrm{~mL}$ ) was added dropwise. The mixture was stirred for $1 \mathrm{~h}$ in a cold ice-bath and then for $1 \mathrm{~h}$ at room temperature until complete formation of epoxysulfone as monitored by TLC. Then, the reaction mixture was cooled at 0 ${ }^{\circ} \mathrm{C}$ and the amine ( $4 \mathrm{mmol}, 4$ equiv.) was slowly added. The reaction mixture was kept for $18 \mathrm{~h}$ at $-8{ }^{\circ} \mathrm{C}$, and then $\mathrm{NaBH}_{4}$ ( 8 mmol, 8 equiv.) was slowly added. The resulting mixture was stirred for $8 \mathrm{~h}$. Then, it was quenched with saturated ammonium chloride aqueous solution $(10 \mathrm{~mL})$ and was allowed to warm up to room temperature and extracted with DCM $(3 \times 10 \mathrm{~mL})$. The organic layers were washed with $1 \mathrm{M}$ hydrochloric acid $(10 \mathrm{~mL})$, saturated sodium bicarbonate aqueous solution $(10 \mathrm{~mL})$, and brine; dried using $\mathrm{Na}_{2} \mathrm{SO}_{4}$; and concentrated under vacuum. The crude mixture was purified by chromatography (silica gel, hexanes/ethyl acetate (7:3 to $1: 1)$ ) to afford the pure compound.

$N^{1}, N^{2}$-Dibenzyl-1-phenylethane-1,2-diamine (2a). Yellowish oil (yield $139 \mathrm{mg}, 44 \%):{ }^{1} \mathrm{H} \mathrm{NMR}\left(300 \mathrm{MHz} \mathrm{CDCl}_{3}\right) \delta$ 7.26-7.07 (m, 15H), 3.71-3.56 (m, 4H), $3.40(\mathrm{~d}, J=13.2 \mathrm{~Hz}$, $1 \mathrm{H}), 2.72-2.65(\mathrm{~m}, 2 \mathrm{H}), 2.21$ (br s, $2 \mathrm{H}) \mathrm{ppm} ;{ }^{13} \mathrm{C} \mathrm{NMR}(75$
$\left.\mathrm{MHz}, \mathrm{CDCl}_{3}\right) \delta 142.50,140.70,140.27,128.66,128.50$, $128.46,128.34,128.22,127.48,127.43,127.07,126.96,61.72$, 56.07, 53.73, $51.42 \mathrm{ppm}$; HRMS (ESI) $\mathrm{m} / z$ calcd for $\mathrm{C}_{22} \mathrm{H}_{24} \mathrm{~N}_{2}[\mathrm{M}+\mathrm{H}]^{+}:$317.2018, found: 317.2015; IR ( $\mathrm{KBr}$ ) $\nu 3421,3281,3062,3029,2957,2921,2864,1630,1497,1462$, $1445,1370,1316,1291,1239,1170,1131,1111,1080,1048$, 1031, 1005, 952, 919, 901, 862, 817, 762, 745, $695 \mathrm{~cm}^{-1}$.

$N^{1}, N^{2}$-Dibenzyl-1-(p-tolyl)ethane-1,2-diamine (2b). Yellowish oil (yield $132 \mathrm{mg}, 40 \%):{ }^{1} \mathrm{H}$ NMR (300 MHz, $\left.\mathrm{CDCl}_{3}\right) \delta 7.23-7.12(\mathrm{~m}, 12 \mathrm{H}), 7.10-7.05(\mathrm{~m}, 2 \mathrm{H}), 3.69-$ $3.61(\mathrm{~m}, 3 \mathrm{H}), 3.62(\mathrm{~d}, J=13.4 \mathrm{~Hz}, 1 \mathrm{H}), 3.42(\mathrm{~d}, J=13.2 \mathrm{~Hz}$, $1 \mathrm{H}), 2.73-2.64(\mathrm{~m}, 2 \mathrm{H}), 2.26$ (s, 3H), 2.16 (br s, $2 \mathrm{H}) \mathrm{ppm}$; ${ }^{13} \mathrm{C} \mathrm{NMR}\left(75 \mathrm{MHz}, \mathrm{CDCl}_{3}\right) \delta 140.67,140.23,139.32,136.96$, $129.31,128.43,128.39,128.29,128.17,128.14,127.32,127.00$, 126.88, 61.33, 56.06, 53.70, 51.31, 21.18 ppm; HRMS (ESI) $m / z$ calcd for $\mathrm{C}_{23} \mathrm{H}_{26} \mathrm{~N}_{2}[\mathrm{M}+\mathrm{H}]^{+}:$331.2174, found: 331.2169; IR (KBr) $\nu$ 3329, 3062, 3027, 2922, 2834, 1604, $1509,1455,1350,1223,1158,1122,1054,837,742,700 \mathrm{~cm}^{-1}$.

$N^{1}, N^{2}$-Dibenzyl-1-(4-chlorophenyl)ethane-1,2-diamine (2c). Yellowish oil (yield $182 \mathrm{mg}, 52 \%):{ }^{1} \mathrm{H}$ NMR (400 MHz, $\left.\mathrm{CDCl}_{3}\right) \delta 7.30-7.14(\mathrm{~m}, 14 \mathrm{H}), 3.70-3.63(\mathrm{~m}, 3 \mathrm{H}), 3.61(\mathrm{~d}, J$ $=13.6 \mathrm{~Hz}, 1 \mathrm{H}), 3.41(\mathrm{~d}, J=13.3 \mathrm{~Hz}, 1 \mathrm{H}), 2.75-2.60(\mathrm{~m}, 2 \mathrm{H})$, 1.70 (br s, $2 \mathrm{H}$ ) ppm; ${ }^{13} \mathrm{C} \mathrm{NMR} \mathrm{(101} \mathrm{MHz,} \mathrm{CDCl}_{3}$ ) $\delta$ 141.05, $140.42,140.19,128.86,128.49,128.41,128.39,128.16,128.04$, 127.16, 127.00, 126.91, 66.74, 63.11, 61.11, 55.99, 53.70, $51.32,51.16$ ppm; HRMS (ESI) $m / z$ calcd for $\mathrm{C}_{22} \mathrm{H}_{23} \mathrm{ClN}_{2}[\mathrm{M}$ $+\mathrm{H}]^{+}$: 351.1628, found: 351.1634; IR (KBr) L 3310, 3061, 3026, 2920, 2831, 1755, 1603, 1494, 1454, 1361, 1204, 1126, $1073,1029,746,701 \mathrm{~cm}^{-1}$.

$N^{1}, N^{2}$-Dibenzyl-1-(4-fluorophenyl)ethane-1,2-diamine (2d). Yellowish oil (yield $100 \mathrm{mg}, 30 \%):{ }^{1} \mathrm{H}$ NMR (400 MHz, $\left.\mathrm{CDCl}_{3}\right) \delta 7.28-7.12(\mathrm{~m}, 12 \mathrm{H}), 7.02-6.92(\mathrm{~m}, 2 \mathrm{H}), 3.69-$ $3.63(\mathrm{~m}, 3 \mathrm{H}), 3.61(\mathrm{~d}, J=12.7 \mathrm{~Hz}, 1 \mathrm{H}), 3.41(\mathrm{~d}, J=13.3 \mathrm{~Hz}$, $1 \mathrm{H}), 2.75-2.61(\mathrm{~m}, 2 \mathrm{H}), 1.77$ (br s, $2 \mathrm{H}) \mathrm{ppm} ;{ }^{13} \mathrm{C} \mathrm{NMR}(101$ $\left.\mathrm{MHz}, \mathrm{CDCl}_{3}\right) \delta 162.06(\mathrm{~d}, J=244.9 \mathrm{~Hz}), 140.52,140.30$, $138.17(\mathrm{~d}, J=3.0 \mathrm{~Hz}), 128.84(\mathrm{~d}, J=7.8 \mathrm{~Hz}), 128.48,128.40$, $128.37,128.16,128.04,126.97,126.89,115.30$ (d, $J=21.1$ $\mathrm{Hz}$ ), 61.06, 56.16, 53.72, 51.31 ppm; HRMS (ESI) $\mathrm{m} / z$ calcd for $\mathrm{C}_{22} \mathrm{H}_{23} \mathrm{FN}_{2}[\mathrm{M}+\mathrm{H}]^{+}$, found 335.1913: 335.1912; IR $(\mathrm{KBr}) \nu$ 3428, 3062, 3027, 2923, 2848, 1649, 1612, 1493, $1455,1421,1351,1092,1037,1015,828,743,701 \mathrm{~cm}^{-1}$.

$N^{1}, N^{2}$-Dibenzyl- $N^{1}$-methyl-1-phenylethane-1,2-diamine (2e). Yellowish oil (yield $135 \mathrm{mg}, 41 \%):{ }^{1} \mathrm{H}$ NMR $(300 \mathrm{MHz}$, $\left.\mathrm{CDCl}_{3}\right) \delta 7.46-7.20(\mathrm{~m}, 15 \mathrm{H}), 3.98-3.84(\mathrm{~m}, 3 \mathrm{H}), 3.58(\mathrm{~d}, J$ $=13.3 \mathrm{~Hz}, 1 \mathrm{H}), 3.38-3.23(\mathrm{~m}, 2 \mathrm{H}), 3.20($ br s, $1 \mathrm{H}), 2.92(\mathrm{dd}$, $J=11.9,5.7 \mathrm{~Hz}, 1 \mathrm{H}), 2.11(\mathrm{~s}, 3 \mathrm{H}) \mathrm{ppm} ;{ }^{13} \mathrm{C} \mathrm{NMR}(75 \mathrm{MHz}$, $\left.\mathrm{CDCl}_{3}\right) \delta 139.58,137.29,128.91,128.81,128.50,128.47$, 128.32 , 128.27, 128.21, 127.60, 127.14, 126.96, 66.63, 58.59, $53.59,49.77,37.48 \mathrm{ppm}$; HRMS (ESI) $\mathrm{m} / z$ calcd for $\mathrm{C}_{23} \mathrm{H}_{26} \mathrm{~N}_{2}[\mathrm{M}+\mathrm{H}]^{+}$: 331.2174, found: 331.2167; IR (KBr) $\nu 3416,3060,3026,2931,2858,1656,1603,1496,1455,1385$, $751,702 \mathrm{~cm}^{-1}$.

$N^{1}, N^{2}$-Dibenzyl-N $N^{1}$-methyl-1-(p-tolyl)ethane-1,2-diamine (2f). Yellowish oil (yield $127 \mathrm{mg}, 37 \%):{ }^{1} \mathrm{H}$ NMR $(300 \mathrm{MHz}$, $\left.\mathrm{CDCl}_{3}\right) \delta 7.27-7.13(\mathrm{~m}, 12 \mathrm{H}), 7.10-6.99(\mathrm{~m}, 2 \mathrm{H}), 3.83-$ $3.76(\mathrm{~m}, 2 \mathrm{H}), 3.73(\mathrm{~d}, J=13.3 \mathrm{~Hz}, 1 \mathrm{H}), 3.51$ (br s, $1 \mathrm{H}), 3.44$ $(\mathrm{d}, J=13.3 \mathrm{~Hz}, 1 \mathrm{H}), 3.24-3.08(\mathrm{~m}, 2 \mathrm{H}), 2.78(\mathrm{dd}, J=11.9$, $5.7 \mathrm{~Hz}, 1 \mathrm{H}), 2.27(\mathrm{~s}, 3 \mathrm{H}), 1.97(\mathrm{~s}, 3 \mathrm{H}) \mathrm{ppm} ;{ }^{13} \mathrm{C} \operatorname{NMR}(75$ $\left.\mathrm{MHz}, \mathrm{CDCl}_{3}\right) \delta 139.61,139.40,137.25,133.94,128.92$, $128.84,128.52$, 128.44, 128.33, 127.19, 126.96, 66.13, 58.56, 53.51, 49.71, 37.42, $21.17 \mathrm{ppm}$; HRMS (ESI) $\mathrm{m} / z$ calcd for $\mathrm{C}_{24} \mathrm{H}_{28} \mathrm{~N}_{2}[\mathrm{M}+\mathrm{H}]^{+}$: 345.2331, found: 345.2328; IR (KBr) $\nu$ 13C NMR (400 MHz) $\delta 3433,3062,3028,2923,2849,2796$, 
$1672,1645,1604,1510,1454,1368,1226,1160,1016,837$, $740,701 \mathrm{~cm}^{-1}$.

$N^{1}, N^{2}$-Dibenzyl-1-(4-chlorophenyl)- $N^{1}$-methylethane-1,2diamine (2g). Yellowish oil (yield $145 \mathrm{mg}, 40 \%$ ): ${ }^{1} \mathrm{H}$ NMR $\left(400 \mathrm{MHz}, \mathrm{CDCl}_{3}\right) \delta 7.35-7.14(\mathrm{~m}, 12 \mathrm{H}), 7.12-7.04(\mathrm{~m}$, $2 \mathrm{H}), 3.86-3.75(\mathrm{~m}, 3 \mathrm{H}), 3.42(\mathrm{~d}, J=13.3 \mathrm{~Hz}, 1 \mathrm{H}), 3.22(\mathrm{~d}, J$ $=13.3 \mathrm{~Hz}, 1 \mathrm{H}), 3.18(\mathrm{~s}, 1 \mathrm{H}), 3.14(\mathrm{dd}, J=12.0,8.9 \mathrm{~Hz}, 1 \mathrm{H})$, $2.80(\mathrm{dd}, J=12.0,5.7 \mathrm{~Hz}, 1 \mathrm{H}), 1.97(\mathrm{~s}, 3 \mathrm{H}) \mathrm{ppm}$; HRMS (ESI) $m / z$ calcd for $\mathrm{C}_{23} \mathrm{H}_{25} \mathrm{ClN}_{2}[\mathrm{M}+\mathrm{H}]^{+}: 365.1788$, found: 365.1788.; ${ }^{13} \mathrm{C}$ NMR (101 MHz, $\left.\mathrm{CDCl}_{3}\right) \delta 139.10,135.51$, 133.47, 130.11, 128.77, 128.63, 128.60, 128.56, 128.42, 128.39, 128.38, 127.43, 127.11, 65.63, 58.59, 53.29, 49.27, 37.34 ppm; IR $(\mathrm{KBr}) \nu 3345,3061,3027,2926,2846,2795,1674,1638$, $1603,1495,1454,1368,1124,1077,1025,745,702 \mathrm{~cm}^{-1}$.

$N^{1}, N^{2}$-Dibenzyl-1-(4-fluorophenyl)- $N^{1}$-methylethane-1,2diamine (2h). Yellowish oil (yield $125 \mathrm{mg}, 36 \%$ ): ${ }^{1} \mathrm{H}$ NMR $\left(300 \mathrm{MHz}, \mathrm{CDCl}_{3}\right) \delta 7.27-7.08(\mathrm{~m}, 12 \mathrm{H}), 7.01-6.90(\mathrm{~m}$, $2 \mathrm{H}), 3.83-3.69(\mathrm{~m}, 3 \mathrm{H}), 3.42(\mathrm{~d}, J=13.3 \mathrm{~Hz}, 1 \mathrm{H}), 3.34$ (br s, $1 \mathrm{H}), 3.24-3.07(\mathrm{~m}, 2 \mathrm{H}), 2.77$ (dd, $J=11.9,5.8 \mathrm{~Hz}, 1 \mathrm{H}), 1.96$ $(\mathrm{s}, 3 \mathrm{H}) \mathrm{ppm} ;{ }^{13} \mathrm{C}$ NMR $\left(75 \mathrm{MHz}, \mathrm{CDCl}_{3}\right) \delta 162.23(\mathrm{~d}, J=$ $245.8 \mathrm{~Hz}), 139.32,139.29,133.05(\mathrm{~d}, J=3.3 \mathrm{~Hz}), 130.31$ (d, $J$ $=7.9 \mathrm{~Hz}), 128.77,128.54,128.38,128.29,127.25,127.06$, 115.07 (d, $J=21.0 \mathrm{~Hz}), 65.80,58.57,53.51,49.75,37.38 \mathrm{ppm}$; HRMS (ESI) $m / z$ calcd for $\mathrm{C}_{23} \mathrm{H}_{25} \mathrm{FN}_{2}[\mathrm{M}+\mathrm{H}]^{+}:$349.2080, found: 349.2078; IR (KBr) $\nu$ 3367, 3062, 3027, 2924, 2850, 2796, 1659, 1596, 1494, 1455, 1396, 1093, 1016, 828, 741, 701 $\mathrm{cm}^{-1}$.

Method B. $t$-BuONa ( $1.2 \mathrm{mmol}, 1.2$ equiv.) was added to an ice-cold solution of chloromethyl phenyl sulfone $(1.2 \mathrm{mmol}$, 1.2 equiv.) in THF $(5 \mathrm{~mL})$. The mixture was stirred for 15 min; then, the corresponding aldehyde ( $1 \mathrm{mmol}, 1$ equiv.) was slowly added as a solution in THF $(5 \mathrm{~mL})$. The reaction mixture was then stirred for $1 \mathrm{~h}$ at $0{ }^{\circ} \mathrm{C}$ and for $1 \mathrm{~h}$ at room temperature. When TLC showed complete formation of epoxysulfone, the reaction was cooled at $-8{ }^{\circ} \mathrm{C}$ and the corresponding amine ( $4 \mathrm{mmol}, 4$ equiv.) was added followed by addition of $\mathrm{LiCl}(10 \mathrm{mmol}, 10$ equiv. $)$. The reaction mixture was kept for $18 \mathrm{~h}$ at $-8{ }^{\circ} \mathrm{C}$. Then, $\mathrm{NaBH}_{4}(8 \mathrm{mmol}, 8$ equiv.) was added portionwise, and the resulting mixture was stirred for additional $8 \mathrm{~h}$. Finally, the reaction mixture was quenched with saturated ammonium chloride aqueous solution $(10 \mathrm{~mL})$ and was then allowed to warm up to room temperature. Then, it was extracted with DCM $(3 \times 10$ $\mathrm{mL})$. The resulting organic layers were washed with $1 \mathrm{M}$ hydrochloric acid $(10 \mathrm{~mL})$, then with sodium bicarbonate saturated aqueous solution $(10 \mathrm{~mL})$, and finally with brine. Washed organic portion was dried using $\mathrm{Na}_{2} \mathrm{SO}_{4}$ and concentrated under vacuum. The resulting crude material was purified by chromatography (silica gel, hexanes/ethyl acetate $(7: 3$ to $1: 1))$ to give the desired compound.

$N^{1}, N^{2}$-Dibutyl-1-phenylethane-1,2-diamine (2i). Yellow oil (yield $76 \mathrm{~g}, 31 \%):{ }^{1} \mathrm{H} \mathrm{NMR}\left(500 \mathrm{MHz}, \mathrm{CDCl}_{3}\right) \delta 7.35-7.23$ $(\mathrm{m}, 5 \mathrm{H}), 3.80$ (dd, $J=7.6,6.3 \mathrm{~Hz}, 1 \mathrm{H}), 3.45$ (br s, $2 \mathrm{H}), 2.91-$ $2.83(\mathrm{~m}, 2 \mathrm{H}), 2.68(\mathrm{qt}, J=11.6,7.3 \mathrm{~Hz}, 2 \mathrm{H}), 2.51-2.39(\mathrm{~m}$, $2 \mathrm{H}), 1.55-1.40(\mathrm{~m}, 4 \mathrm{H}), 1.37-1.24(\mathrm{~m}, 4 \mathrm{H}), 0.89(\mathrm{t}, J=7.4$ $\mathrm{Hz}, 3 \mathrm{H}), 0.85(\mathrm{t}, J=7.3 \mathrm{~Hz}, 3 \mathrm{H}) \mathrm{ppm} ;{ }^{13} \mathrm{C} \mathrm{NMR}(126 \mathrm{MHz}$, $\left.\mathrm{CDCl}_{3}\right) \delta 141.66,128.63,128.49,127.22,127.06,62.00,55.42$, 48.93, 47.10, 32.03, 31.28, 20.38, 20.27, 13.87, $13.81 \mathrm{ppm}$; HRMS (ESI) $m / z$ calcd for $\mathrm{C}_{16} \mathrm{H}_{28} \mathrm{~N}_{2}[\mathrm{M}+\mathrm{H}]^{+}:$249.2331, found: 249.2329; IR (KBr) $\nu$ 3411, 3061, 3029, 2961, 2929, 2869, 1653, 1602, 1552, 1458, 1385, 762, $704 \mathrm{~cm}^{-1}$.

1-Phenyl- $N^{1}, N^{2}$-bis(3-phenylpropyl)ethane-1,2-diamine (2j). Yellow oil (yield $144 \mathrm{~g}, 39 \%$ ): ${ }^{1} \mathrm{H}$ NMR (500 MHz,
$\left.\mathrm{CDCl}_{3}\right) \delta 7.42-7.14(\mathrm{~m}, 15 \mathrm{H}), 3.78(\mathrm{dd}, J=8.6,5.1 \mathrm{~Hz}, 1 \mathrm{H})$, $2.90-2.79(\mathrm{~m}, 2 \mathrm{H}), 2.77-2.61(\mathrm{~m}, 8 \mathrm{H}), 2.61-2.51(\mathrm{~m}, 2 \mathrm{H})$, 1.93-1.77 (m, 4H) ppm; ${ }^{13} \mathrm{C}$ NMR $\left(126 \mathrm{MHz}, \mathrm{CDCl}_{3}\right) \delta$ $142.34,142.21,141.91,128.58,128.41,128.32,127.39,127.22$, $125.86,125.74,62.50,56.11,48.97,47.03,33.60,33.50,31.77$, 31.28 ppm; HRMS (ESI) $m / z$ calcd for $\mathrm{C}_{26} \mathrm{H}_{32} \mathrm{~N}_{2}[\mathrm{M}+\mathrm{H}]^{+}$: 373.2644, found: 373.2641; IR (KBr) $\nu$ 3164, 3068, 3034, 2994, 1583, 1494, 1478, 1460, 1449, 1401, 1326, 1314, 1288, $1232,1192,1181,1166,1152,1087,1077,1059,1025,999$, 982, 936, 909, 857, 809, 786, 762, 742, 716, 700, $687 \mathrm{~cm}^{-1}$.

2-Phenylpiperazine (3a). White crystals, mp $115-119{ }^{\circ} \mathrm{C}^{14}$ (yield $49 \mathrm{mg}, 30 \%$ ): ${ }^{1} \mathrm{H}$ NMR (500 MHz, $\mathrm{CDCl}_{3}$ ) $\delta 7.40-7.32$ (m, 5H), $3.79(\mathrm{dd}, J=11.3,2.8 \mathrm{~Hz}, 1 \mathrm{H}), 3.54($ br s, $1 \mathrm{H})$, $3.33-3.21(\mathrm{~m}, 2 \mathrm{H}), 3.00(\mathrm{td}, J=12.5,2.7 \mathrm{~Hz}, 1 \mathrm{H}), 2.89-2.76$ $(\mathrm{m}, 1 \mathrm{H}), 2.67(\mathrm{dt}, J=13.3,11.5 \mathrm{~Hz}, 1 \mathrm{H}), 1.69(\mathrm{br} \mathrm{s}, 2 \mathrm{H})$ ppm; ${ }^{13} \mathrm{C}$ NMR (101 MHz, $\left.\mathrm{CDCl}_{3}\right) \delta 140.0,128.9,128.2$, $126.5,60.7,59.0,52.3,46.4$ ppm; HRMS (ESI) $\mathrm{m} / z$ calcd for $\mathrm{C}_{10} \mathrm{H}_{14} \mathrm{~N}_{2}[\mathrm{M}+\mathrm{H}]^{+}:$163.1233, found: 163.1235; IR (KBr) $\nu$ 3166, 3092, 3071, 3059, 3035, 3002, 2232, 1918, 1904, 1800, $1602,1584,1493,1480,1451,1422,1386,1324,1311,1287$, $1267,1227,1189,1161,1147,1115,1102,1088,1070,1056$, $1041,1025,1013,999,928,909,855,834,808,790,749,721$, $704,682 \mathrm{~cm}^{-1}$.

2-Methyl-3-(p-tolyl)piperazine (3b). White crystals, $\mathrm{mp}$ 161-163 ${ }^{\circ} \mathrm{C}^{15}$ (yield $60 \mathrm{mg}, 34 \%$ ): ${ }^{1} \mathrm{H}$ NMR (400 MHz, $\left.\mathrm{CDCl}_{3}\right) \delta 7.16(\mathrm{~d}, J=8.1 \mathrm{~Hz}, 1 \mathrm{H}), 7.09(\mathrm{~d}, J=7.9 \mathrm{~Hz}, 1 \mathrm{H})$, $3.66(\mathrm{dd}, J=11.2,2.7 \mathrm{~Hz}, 1 \mathrm{H}), 3.29$ (br s, $1 \mathrm{H}), 3.22-3.12(\mathrm{~m}$, $2 \mathrm{H}), 2.91(\mathrm{td}, J=12.4,2.6 \mathrm{~Hz}, 1 \mathrm{H}), 2.74(\mathrm{ddd}, J=25.2,12.4$, $3.3 \mathrm{~Hz}, 1 \mathrm{H}), 2.59(\mathrm{dt}, J=13.2,11.6 \mathrm{~Hz}, 1 \mathrm{H}), 2.27(\mathrm{~s}, 3 \mathrm{H})$, 1.47 (br s, $2 \mathrm{H}) \mathrm{ppm} ;{ }^{13} \mathrm{C} \mathrm{NMR}\left(101 \mathrm{MHz}, \mathrm{CDCl}_{3}\right) \delta 138.3$, 138.2, 129.5, 126.8, 60.5, 59.0, 52.3, 46.2, 21.1 ppm; HRMS (ESI) $m / z$ calcd for $\mathrm{C}_{11} \mathrm{H}_{17} \mathrm{~N}_{2}[\mathrm{M}+\mathrm{H}]^{+}: 177.1390$, found: 177.1392; IR (KBr) $\nu$ 3283, 3150, 3055, 3026, 2871, 2435, 2390, 2311, 2265, 2161, 2033, 1977, 1769, 1600, 1519, 1493, $1327,1313,1211,1165,1130,1074,1042,1022,1006,953$, $895,859,832,801 \mathrm{~cm}^{-1}$.

2-Phenylquinoxaline (4). Orange crystals, $\mathrm{mp} 69-70{ }^{\circ} \mathrm{C}^{16}$ (yield $121 \mathrm{mg}, 59 \%):{ }^{1} \mathrm{H}$ NMR $\left(300 \mathrm{MHz}, \mathrm{CDCl}_{3}\right) \delta 9.22(\mathrm{~s}$, $1 \mathrm{H}), 8.13-7.97(\mathrm{~m}, 4 \mathrm{H}), 7.71-7.57(\mathrm{~m}, 2 \mathrm{H}), 7.49-7.38(\mathrm{~m}$, $3 \mathrm{H}) \mathrm{ppm} ;{ }^{13} \mathrm{C}$ NMR $\left(75 \mathrm{MHz}, \mathrm{CDCl}_{3}\right) \delta 151.86,143.39$, 142.32, 141.60, 136.80, 130.31, 130.22, 129.66, 129.57, 129.18, $129.15,127.58$ ppm; HRMS (ESI) $m / z$ calcd for $\mathrm{C}_{14} \mathrm{H}_{14} \mathrm{~N}_{2}[\mathrm{M}$ $+\mathrm{H}]^{+}$: 207.0922, found: 207.0922; IR (KBr) $\nu \delta 3088,3075$, 3066, 2998, 1908, 1606, 1582, 1513, 1478, 1449, 1434, 1405, $1392,1326,1314,1292,1273,1236,1191,1181,1168,1154$, $1101,1086,1074,1059,1026,1014,999,952,937,916,865$, $846,802,769,757,719,688 \mathrm{~cm}^{-1}$.

\section{ASSOCIATED CONTENT}

\section{Supporting Information}

The Supporting Information is available free of charge on the ACS Publications website at DOI: 10.1021/acsomega. 8 b03030.

Graphical NMR spectra of all compounds: ${ }^{1} \mathrm{H}$ NMR spectrum of 1a (Figure S1); ${ }^{13} \mathrm{C}$ NMR spectrum of 1a (Figure S2); ${ }^{1} \mathrm{H}$ NMR spectrum of $2 \mathrm{a}$ (Figure S3); ${ }^{13} \mathrm{C}$ NMR spectrum of $2 \mathrm{a}$ (Figure S4); ${ }^{1} \mathrm{H}$ NMR spectrum of $\mathbf{2 b}$ (Figure S5); ${ }^{13} \mathrm{C}$ NMR spectrum of $\mathbf{2 b}$ (Figure S6); ${ }^{1} \mathrm{H}$ NMR spectrum of $2 \mathrm{c}$ (Figure S7); ${ }^{13} \mathrm{C}$ NMR spectrum of $2 \mathrm{c}$ (Figure S8); ${ }^{1} \mathrm{H}$ NMR spectrum of $2 \mathrm{~d}$ (Figure S9); ${ }^{13} \mathrm{C}$ NMR spectrum of 2d (Figure S10); ${ }^{1} \mathrm{H}$ NMR spectrum of $2 \mathrm{e}$ (Figure S11); ${ }^{13} \mathrm{C}$ NMR spectrum 
of $2 \mathbf{e}$ (Figure $\mathrm{S} 12) ;{ }^{1} \mathrm{H}$ NMR spectrum of $2 \mathrm{f}$ (Figure $\mathrm{S} 13) ;{ }^{13} \mathrm{C}$ NMR spectrum of $2 \mathrm{f}$ (Figure S14); ${ }^{1} \mathrm{H}$ NMR spectrum of $2 \mathrm{~g}$ (Figure $\mathrm{S} 15$ ); ${ }^{13} \mathrm{C}$ NMR spectrum of $2 \mathrm{~g}$ (Figure S16); ${ }^{1} \mathrm{H}$ NMR spectrum of $\mathbf{2 h}$ (Figure S17); ${ }^{13} \mathrm{C}$ NMR spectrum of $2 \mathrm{~h}$ (Figure S18); ${ }^{1} \mathrm{H}$ NMR spectrum of $2 \mathbf{i}$ (Figure S19); ${ }^{13} \mathrm{C}$ NMR spectrum of $2 \mathbf{i}$ (Figure S20); ${ }^{1} \mathrm{H}$ NMR spectrum of $2 \mathrm{j}$ (Figure S21); ${ }^{13} \mathrm{C}$ NMR spectrum of $2 \mathrm{j}$ (Figure S22); ${ }^{1} \mathrm{H}$ NMR spectrum of $3 a$ (Figure S23); ${ }^{13} \mathrm{C}$ NMR spectrum of $3 a$ (Figure S24); ${ }^{1} \mathrm{H}$ NMR spectrum of $\mathbf{3 b}$ (Figure S25); ${ }^{13} \mathrm{C}$ NMR spectrum of $3 \mathbf{b}$ (Figure S26); ${ }^{1} \mathrm{H}$ NMR spectrum of 4 (Figure S27); ${ }^{13} \mathrm{C}$ NMR spectrum of 4 (Figure S28) (PDF)

\section{AUTHOR INFORMATION}

\section{Corresponding Author}

*E-mail: fgonzale@uji.es.

\section{ORCID}

Florenci V. González: 0000-0001-5709-734X

Notes

The authors declare no competing financial interest.

\section{ACKNOWLEDGMENTS}

This work was financially supported by Generalitat Valenciana (AICO/2016/32). L.B.-I. thanks Ministerio de Ciencia, Innovación y Universidades for funding a Ph.D. fellowship (FPU17/06209) and also Universitat Jaume I (PREDOC/ 2017/25). We also thank Serveis Centrals d'Instrumentació Cientifica of Universitat Jaume I for the technical support.

\section{REFERENCES}

(1) (a) Michalson, E. T.; Szmuszkovicz, J. Medicinal agents incorporating the 1,2-diamine functionality. Prog. Drug Res. 1989, 33, 135-149. (b) Lucet, D.; Gall, T. L.; Mioskowski, C. The Chemistry of Vicinal Diamines. Angew. Chem., Int. Ed. 1998, 37, 2580-2627. (c) Saibabu Kotti, S. R. S.; Timmons, C.; Li, G. Vicinal Diamino Functionalities as Privileged Structural Elements in Biologically Active Compounds and Exploitation of their Synthetic Chemistry. Chem. Biol. Drug Des. 2006, 67, 101-114. (d) Desai, M. C.; Lefkowitz, S. L.; Thadeio, P. F.; Longo, K. P.; Snider, R. M. Discovery of a potent substance $\mathrm{P}$ antagonist: recognition of the key molecular determinant. J. Med. Chem. 1992, 35, 4911-4913. (e) Hanessian, S.; Bayrakdarian, M.; Luo, X. Total Synthesis of A315675: A Potent Inhibitor of Influenza Neuraminidase. J. Am. Chem. Soc. 2002, 124, 4716-4721.

(2) (a) Shigematsu, N.; Setoi, H.; Uchida, I.; Shibata, T.; Terano, H.; Hashimoto, M. Structure and synthesis of FR900490, a new immunomodulating peptide isolated from a fungus. Tetrahedron Lett. 1988, 29, 5147-5150. (b) Kinnel, R. B.; Gehrken, H.-P.; Scheuer, P. J. Palau'amine: a cytotoxic and immunosuppressive hexacyclic bisguanidine antibiotic from the sponge Stylotella agminata. J. Am. Chem. Soc. 1993, 115, 3376-3377. (c) Kuwahara, A.; Nishikiori, T.; Shimada, N.; Nakagawa, T.; Fukazawa, H.; Mizuno, S.; Uehara, Y. NA22598A(1), a novel antitumor substance produced by Streptomyces sp. NA22598. J. Antibiot. 1997, 50, 712-713. (d) Nishimura, S.; Matsunaga, S.; Shibazaki, M.; Suzuki, K.; Furihata, K.; van Soest, R. W. M.; Fusetani, N. Massadine, a novel geranylgeranyltransferase type I inhibitor from the marine sponge Stylissa aff. massa. Org. Lett. 2003, 5, 2255-2257. (e) Tsuritani, N.; Yamada, K.; Yoshikawa, N.; Shibasaki, M. Catalytic asymmetric syntheses of ICI-199441 and CP-99994 using nitro-Mannich reaction. Chem. Lett. 2002, 276-277.

(3) (a) Surry, D. S.; Buchwald, S. L. Diamine ligands in coppercatalyzed reactions. Chem. Sci. 2010, 1, 13-31. (b) Dong, Z.; Bai, S.; Yap, G. P. A.; Fox, J. M. Interplay between the diamine structure and absolute helicity in Ni-salen metallofoldamers. Chem. Commun. 2011, 47, 3781-3783.

(4) (a) Hazeldine, S. T.; Polin, L.; Kushner, J.; Paluch, J.; White, K.; Edelstein, M.; Palomino, E.; Corbett, T. H.; Horwitz, J. P. Design, synthesis, and biological evaluation of analogues of the antitumor agent, 2-\{4-[(7-chloro-2-quinoxalinyl)oxy]phenoxy\}propionic acid (XK469). J. Med. Chem. 2001, 44, 1758-1776. (b) Kong, D.; Park, E. J.; Stephen, A. G.; Calvani, M.; Cardellina, J. H.; Monks, A.; Fisher, R. J.; Shoemaker, R. H.; Melillo, G. Echinomycin, a small-molecule inhibitor of hypoxia-inducible factor-1 DNA-binding activity. Cancer Res. 2005, 65, 9047-9055.

(5) (a) el-Ashry, E. S.; Abdel-Rahman, A. A. H.; Rashed, N.; Rasheed, H. A. Homoacyclovir analogues of unnatural bases and their activity against Hepatitis B virus. Pharmazie 1999, 54, 893-897. (b) You, L.; Cho, E. J.; Leavitt, J.; Mad, L.; Montelione, G. T.; Anslyn, E. V.; Krug, R. M.; Ellington, A.; Robertus, J. D. Synthesis and evaluation of quinoxaline derivatives as potential influenza NS1A protein inhibitors. Bioorg. Med. Chem. Lett. 2011, 21, 3007-3011.

(6) (a) Badran, M. M.; Abonzid, K. A.; Hussein, M. H. Synthesis of certain substituted quinoxalines as antimicrobial agents (part II). Arch. Pharm. Res. 2003, 26, 107-113. (b) Seitz, L. E.; Suling, W. J.; Reynolds, R. C. Synthesis and antimycobacterial activity of pyrazine and quinoxaline derivatives. J. Med. Chem. 2002, 45, 5604-5606.

(7) Abu-Hashem, A. A.; Gouda, M. A.; Badria, F. A. Synthesis of some new pyrimido $\left[2^{\prime}, 1^{\prime}: 2,3\right]$ thiazolo[4,5-b]quinoxaline derivatives as anti-inflammatory and analgesic agents. Eur. J. Med. Chem. 2010, 45, 1976-1981.

(8) (a) Khattab, S. N.; Hassan, S. Y.; Bekhit, A. A.; El Massry, A. M.; Langer, V.; Amer, A. Synthesis of new series of quinoxaline based MAO-inhibitors and docking studies. Eur. J. Med. Chem. 2010, 45, 4479-4489. (b) Srinivas, C.; Kumar, C. N. S. S. P.; Rao, V. J.; Palaniappan, S. Efficient, convenient and reusable polyaniline-sulfate salt catalyst for the synthesis of quinoxaline derivatives. J. Mol. Catal. A: Chem. 2007, 256, 227-230.

(9) (a) Myers, M. R.; He, W.; Hanney, B.; Setzer, N.; Maguire, M. P.; Zulli, A.; Bilder, G.; Galzciniski, H.; Amin, D.; Needle, S.; Spada, A. P. Potent quinoxaline-based inhibitors of PDGF receptor tyrosine kinase activity. Part 1: SAR exploration and effective bioisosteric replacement of a phenyl substituent. Bioorg. Med. Chem. Lett. 2003, 13, 3091-3095. (b) Dietrich, B.; Diederchsen, U. Synthesis of cyclopeptidic analogues of triostin a with quinoxalines or nucleobases as chromophores. Eur. J. Org. Chem. 2005, 2005, 147-153.

(10) (a) MacDonald, M. J.; Schipper, D. J.; Ng, P. J.; Moran, J.; Beauchemin, A. M. A Catalytic Tethering Strategy: Simple Aldehydes Catalyze Intermolecular Alkene Hydroaminations. J. Am. Chem. Soc. 2011, 133, 20100-20103. (b) Zhao, S.-B.; Bilodeau, E.; Lemieux, V.; Beauchemin, A. M. Hydrogen Bonding Directed Intermolecular Cope-Type Hydroamination of Alkenes. Org. Lett. 2012, 14, 50825085. (c) Hesp, C. R.; MacDonald, M. J.; Zahedi, M. M.; Bilodeau, D. A.; Zhao, S.-B.; Pesant, M.; Beauchemin, A. M. Formaldehyde as Tethering Organocatalyst: Highly Diastereoselective Hydroaminations of Allylic Amines. Org. Lett. 2015, 17, 5136-5139.

(11) (a) Agut, J.; Vidal, A.; Rodríguez, S.; González, F. V. Dynamic Kinetic Asymmetric Ring-Opening/Reductive Amination Sequence of Racemic Nitroepoxides with Chiral Amines: Enantioselective Synthesis of Chiral Vicinal Diamines. J. Org. Chem. 2013, 78, 5717-5722. (b) Vidal-Albalat, A.; Rodríguez, S.; González, F. V. Nitroepoxides as Versatile Precursors to 1,4-Diamino Heterocycles. Org. Lett. 2014, 16, 1752-1755. (c) Nosood, Y. L.; Halimehjani, A. Z.; González, F. V. Regioselective Opening of Nitroepoxides with Unsymmetrical Diamines. J. Org. Chem. 2018, 83, 1252-1258.

(12) Hayashi, Y. Pot-economy and one-pot synthesis. Chem. Sci. 2016, 7, 866-880.

(13) Atmospheric oxygen might oxidize the dihydroquinoxaline intermediate to quinoxaline.

(14) (a) Elmaleh, D. R.; Songwoon, C.; Fishman, A. J. Diagnostic and Therapeutics Alkyl Piperidine/Piperazine Compounds and Process. U.S. Patent US7381822B2, 2008. (b) Kitchen, L. J.; 
Pollard, C. B. Derivatives of piperazine. 21. Synthesis of piperazine and C-substituted piperazines. J. Am. Chem. Soc. 1947, 69, 854-855.

(15) Leclerc, J.; Fagnou, K. Palladium-catalyzed cross-coupling reactions of diazine $\mathrm{N}$-oxides with aryl chlorides, bromides, and iodides. Angew. Chem., Int. Ed. 2006, 45, 7781-7786.

(16) Tobisu, M.; Takayuki, F.; Naoto, C. Visible Light-mediated Direct Arylation of Arenes and Heteroarenes Using Diaryliodonium Salts in the Presence and Absence of a Photocatalyst. Chem. Lett. 2013, 42, 1203-1205. 